MESQUITA FILHO, M.V. de; SOUZA, A.F.; MOITA, A.W.; RAMAGEM, R.D. Produção comercializável e teores de Cu e Zn em cenoura em decorrência da ação residual de fósforo e composto de lixo em solo sob cerrado. Horticultura Brasileira, Brasília, v. 20, n. 2, p. 153-157, junho 2.002.

\title{
Produção comercializável e teores de Cu e Zn em cenoura em decorrên- cia da ação residual de fósforo e composto de lixo em solo sob cerrado
}

\author{
Manoel V. de Mesquita Filho'; Antônio F. Souza ${ }^{1}$; Antônio W. Moita; Ricardo D. Ramagem ${ }^{2}$ \\ ${ }^{1}$ Embrapa Hortaliças, C.Postal 218, 70.359-970 Brasília-DF; ${ }^{2}$ SCEN, Trecho 03, Clube do Servidor, Lotes 1A e 1B, $70.800-970$ Brasília \\ -DF; E-mail: mesquita@cnph.embrapa.br
}

\section{RESUMO}

Realizou-se em 1997, em condições de campo um experimento em Latossolo Vermelho Escuro distrófico argiloso sob cerrado de Brasília, para avaliar o efeito residual das aplicações em anos anteriores, a lanço de doses de fósforo (superfosfato triplo), e de composto de lixo na produção de cenoura (Daucus carota), cv. Brasília, assim como nos teores de cobre e zinco em raízes frescas. Aproveitou-se o mesmo delineamento experimental de blocos ao acaso com 3 repetições, no mesmo campo experimental dos experimentos anteriores distribuídos num esquema fatorial 3 x 5 incluindo-se 3 níveis de fósforo $\left(0 ; 400\right.$ e $800 \mathrm{~kg} \mathrm{ha}^{-1}$ de $\left.\mathrm{P}_{2} \mathrm{O}_{5}\right)$ e 5 níveis de composto de lixo $\left(0 ; 20 ; 40 ; 60\right.$ e $\left.80 \mathrm{t} \mathrm{ha}^{-1}\right)$. A colheita foi realizada aos 90 dias após o plantio. A análise estatística dos dados de produção total de raízes revelou efeito residual da adubação dos últimos dois anos em linear e quadrático altamente significativo $(\mathrm{p}<0,01)$ para fósforo $\mathrm{e}$ composto de lixo. A interação linear de $\mathrm{P}$ x quadrática composto de lixo foi altamente significativa $(\mathrm{p}<0,01)$. A produção total máxima de $26,5 \mathrm{tha}^{-1}$, correspondendo a $18,5 \mathrm{tha}^{-1}$ de raízes comercializáveis, foi assegurada pelas doses calculadas de $762,5 \mathrm{~kg} \mathrm{ha}^{-1} \mathrm{de}_{2} \mathrm{O}_{5}$ e 53,2 $\mathrm{t} \mathrm{ha}^{-1}$ de composto de lixo de acordo com a função $\mathrm{Y}(\mathrm{PROD})=$ $4,541143+4,0088 \times 10^{-2} \mathrm{P}_{2} \mathrm{O}_{5}+2,50486 \times 10^{-1} \mathrm{CLx}-2,5619 \times 10$ ${ }^{5}\left(\mathrm{P}_{2} \mathrm{O}_{5}\right)^{2}-1,9125 \times 10^{-5}\left(\mathrm{CLx} * \mathrm{P}_{2} \mathrm{O}_{5}\right)-2,216 \times 10^{-3} \mathrm{CLx}^{2}\left(\mathrm{R}^{2}=\right.$ $0,96)$, onde $\mathrm{CLx}=$ composto de lixo. A aplicação ao solo das doses de composto de lixo alteraram o $\mathrm{pH}$, a condutividade elétrica e os teores de $\mathrm{Cu}$ e $\mathrm{Zn}$. Mesmo nas doses mais elevadas de fósforo e de composto de lixo aplicadas não foram observados efeitos fitotóxicos de $\mathrm{Cu}$ ou de $\mathrm{Zn}$. Os teores desses elementos em raízes in natura estiveram abaixo dos permitidos pela legislação brasileira de alimentos.

Palavras-chave: Daucus carota, resíduo urbano, adubação orgânica, adubação fosfatada, nutrição mineral, produtividade, micronutrientes, metal pesado.

\begin{abstract}
Marketable yield and contents of $\mathrm{Cu}$ and $\mathrm{Zn}$ in carrot as influenced by residual phosphate and urban compost in a cerrado soil
\end{abstract}

A field experiment was conducted on a clayey Yellow Red Oxisol to evaluate the residual effect of the application of phosphorus and urban waste compost of the previous two years on the root production of carrot cv. Brasília. The soil of the previous experiment design used a factorial consisting of three levels of phosphorus $(0 ; 400$ and $800 \mathrm{~kg}$ $\left.\mathrm{ha}^{-1}\right)$, applied as triple superphosphate combined with five levels of urban waste compost $\left(0 ; 20 ; 40 ; 60\right.$ and 80 t.ha $\left.^{-1}\right)$, was arranged in randomized complete blocks with three replicates. Carrot plants were harvested 90 days after planting. After the harvest, a linear and quadratic effect for phosphorus and urban waste compost $(\mathrm{p}<0,01)$ was observed. The linear interaction $P \mathrm{x}$ quadratic urban compost was highly significant $(\mathrm{p}<0,01)$. The maximum root total production was $26.5 \mathrm{t}^{\text {ha }} \mathrm{a}^{-1}$ corresponding to $18.5 \mathrm{tha}^{-1}$ of marketable yield, estimated by the calculated doses of $762.5 \mathrm{~kg} \mathrm{ha}^{-1}$ of $\mathrm{P}_{2} \mathrm{O}_{5}$ and $53.2 \mathrm{tha}{ }^{-1}$ of urban waste compost according to the function: $\mathrm{Y}(\mathrm{PROD})=4.541143$ $+4.0088 \times 10^{-2} \mathrm{P}_{2} \mathrm{O}_{5}+2.50486 \times 10^{-1} \mathrm{CLx}-2.5619 \times 10^{-5}\left(\mathrm{P}_{2} \mathrm{O}_{5}\right)^{2}-$ $1.9125 \times 10^{-5}\left(\mathrm{CLx}^{2} * \mathrm{P}_{2} \mathrm{O}_{5}\right)-2.216 \times 10^{-3} \mathrm{CLx}^{2}\left(\mathrm{R}^{2}=0,96\right)$, where $\mathrm{CLx}=$ urban waste compost. Doses of urban waste compost applied to the soil, affected the values of $\mathrm{pH}$, electric conductivity, and the available contents of $\mathrm{Cu}$ and $\mathrm{Zn}$. Even under the highest levels of phosphorus and urban waste compost applied in the experiment, no phytotoxic effect on carrot plants was observed, and in the edible part, none of the elements reached the maximum tolerant limit for food, as established by the Brazilian legislation.

Keywords: Daucus carota, urban waste, organic fertilizer, phosphate fertilizer, mineral nutrition, productivity, micronutrients, heavy metal.

(Aceito para publicação em 07 de maio de 2.002)

$\mathrm{N}^{\mathrm{o}}$ Distrito Federal (safra de 1997), a produção de cenoura foi de $31.147 \mathrm{t}$ com um rendimento de $23,7 \mathrm{t}$ $\mathrm{ha}^{-1}$, o menor dos últimos 10 anos. As principais regiões produtoras são os núcleos rurais de Brazlândia e Alexandre Gusmão, que juntos representam mais de $80 \%$ da área mencionada.

Segundo o Serviço de Limpeza Urbana $^{1}$, a Unidade Experimental de
Compostagem e Reciclagem de Brazlândia, Distrito Federal (UECRBDF), recebe aproximadamente 30 t.dia ${ }^{-1}$ de lixo, das quais $70 \%$ são destinadas à compostagem.

Nos últimos 5 anos vem aumentando o número de horticultores locais, que utilizam esse composto como fonte alternativa de matéria orgânica em substituição ao esterco de gado ou de gali- nha. Entretanto, face aos metais pesados contidos no composto de lixo, poderá ocorrer contaminação nas raízes de cenoura principalmente após aplicações sucessivas deste insumo (Cravo et al.,1998). Dentre os metais pesados, $\mathrm{Cu}$ e $\mathrm{Zn}$ são citados como perigosos devido à toxicidade e potencial de bioacumulação (He et al.,1992; Costa et al., 1994; Costa et al., 1997; Cravo,

\footnotetext{
${ }^{1}$ Dados fornecidos ao primeiro autor em julho de 1998, pelo Dr. Luciano Sales de Oliveira, Diretor Geral do Serviço de Limpeza Urbana do Distrito Federal.
} 
et al.,1998). A aplicação de $14 \mathrm{t} \mathrm{ha}^{-1} \mathrm{de}$ composto de lixo com $500 \mathrm{~kg} \mathrm{ha}^{-1}$ de um fertilizante N-P-K, na fórmula 15 15-15, para a cultura do sorgo por dois ciclos, não promoveu diferenças de produção entre esses fertilizantes (Cabrera et al.,1989). Contudo, os autores alertam para possíveis problemas com excesso de $\mathrm{Cu}$ e $\mathrm{Zn}$, se doses maiores do composto forem utilizadas ou aplicações sucessivas forem feitas.

Adições maciças de determinadas fontes de matéria orgânica, poderão elevar o $\mathrm{pH}$ e a condutividade elétrica na solução do solo, comprometendo a produção de certas hortaliças. Neste sentido, Bernstein \& Ayres (1953) observaram redução de $50 \%$ na produção de raízes de cenoura quando a condutividade elétrica do solo era de 4,6 $\mathrm{dS} \mathrm{m}^{-1}$.

Com a utilização de resíduos de lixo urbano como fontes de matéria orgânica é necessária a determinação da dose correta para evitar conseqüências indesejáveis ao ambiente. Apesar da determinação dos teores totais de metais não ser um bom critério para avaliar a contaminação do solo com metais pesados, ela tem sido utilizada porque ainda não foram selecionados extratores adequados para a determinação dos teores disponíveis para plantas (Alves et al., 1999).

No Brasil, as informações sobre absorção de metais pesados por plantas olerícolas, cultivadas em solos tratados com compostos orgânicos urbanos, são ainda bastante escassas. Para o cultivo da cenoura, foram encontrados nos últimos 15 anos na literatura nacional, apenas quatro publicações (Mesquita Filho et al., 1985; Fortes Neto et al., 1996; Pérez et al., 1996; Costa et al., 1997). Destes apenas o último relata teores de $\mathrm{Cu}, \mathrm{Zn}$ e $\mathrm{Cd}$ nessa hortaliça, em decorrência da aplicação de doses de composto de lixo urbano.

Mesquita Filho et al. (1985), realizaram no Distrito Federal um experimento em Latossolo Vermelho-Escuro distrófico (LVd), argiloso, onde a aplicação de $30 \mathrm{t} \mathrm{ha}^{-1}$ de composto de lixo associado às doses de 138 e $731 \mathrm{~kg} \mathrm{ha}^{-1}$ de nitrogênio e fósforo respectivamente, proporcionou 41,4 t.ha ${ }^{-1}$ de cenoura comercial cv. Nantes, considerada produção normal para o Distrito Federal, naquela época.
Com o presente estudo visou-se avaliar o efeito residual da aplicação a lanço do fósforo e do composto de lixo em um terceiro cultivo, além dos teores totais de $\mathrm{Cu}$ e $\mathrm{Zn}$ nessa olerácea. As doses de fósforo foram aplicadas apenas no primeiro cultivo da cenoura enquanto, as de composto de lixo no primeiro e no segundo, como procede a maioria dos olericultores locais. Vale ressaltar que não é rara a aplicação de matéria orgânica em três cultivos sucessivos de cenoura.

\section{MATERIAL E MÉTODOS}

O experimento foi realizado em Latossolo Vermelho Escuro distrófico $(\mathrm{LVd})$ argiloso em área experimental da Embrapa Hortaliças, em Brasília (DF). O delineamento foi de blocos ao acaso com 3 repetições e os tratamentos distribuídos num esquema fatorial $3 \times 5$ com 3 níveis de fósforo $(0 ; 400$ e 800 $\mathrm{kg} \mathrm{ha}^{-1}$ de $\left.\mathrm{P}_{2} \mathrm{O}_{5}\right)$ e 5 níveis de composto de lixo $\left(0 ; 20 ; 40 ; 60\right.$ e 80 t ha $\left.^{-1}\right)$, incorporados mecanicamente ao solo até a profundidade de $20 \mathrm{~cm}$ um mês antes da primeira semeadura da cenoura cv. Brasília. A disposição e localização das parcelas foi a mesma nos três anos de execução do experimento.

Cada repetição foi constituída por 4 canteiros de 0,15 $\mathrm{m}$ de altura e $1 \mathrm{~m}$ de largura, com 4 fileiras de plantas. A parcela $(4 \mathrm{~m} \mathrm{x} 1 \mathrm{~m})$ foi semeada no espaçamento de $0,25 \mathrm{~m} \times 0,05 \mathrm{~m}$, de modo a conter 16 fileiras de plantas, sendo as áreas total e útil de $16 \mathrm{~m}^{2}$ e 7,2 $\mathrm{m}^{2}$ respectivamente. Os teores de $\mathrm{Cu}$ e $\mathrm{Zn}$ em raízes in natura foram comparados com os permitidos pela legislação brasileira de alimentos (ABIA, 1985).

Ao todo foram feitos três cultivos (04/ 05/95 a 03/08/95; 23/10/95 a 21/01/96 e 03/04/97 a 04/07/97). Antes de cada cultivo, o solo em cada parcela era revolvido e nivelado com enxada. Utilizandose um trado contendo $30 \mathrm{~mm}$ de diâmetro coletaram-se três subamostragens na profundidade $0-20 \mathrm{~cm}$ por parcela para compor uma amostra de solo. Por ocasião da terceira semeadura da cenoura, os valores médios de $\mathrm{pH}$ nos tratamentos $0 ; 20 ; 40 ; 60$ e 80 t ha $^{-1}$ de composto de lixo eram 5,$44 ; 5,49 ; 5,52 ; 5,54$ e 5,60 e os de condutividade elétrica 0,$03 ; 0,1$; 0,$2 ; 0,4$ e $0,4 \mathrm{dS} \mathrm{m}^{-1}$. Nesta data, os teo- res médios das análises químicas do solo das parcelas testemunhas de fósforo e composto de lixo segundo os métodos da Embrapa (1979) foram: $\mathrm{pH}$ em $\mathrm{H}_{2} \mathrm{O}$ $(1: 2,5)=5,44 ; \mathrm{Al}^{3+}=0,38 \mathrm{cmol} \mathrm{kg}^{-1}$; $\mathrm{Ca}^{+2}=1,96 \mathrm{cmol}_{\mathrm{c}} \mathrm{kg}^{-1} ; \mathrm{Mg}^{+2}=0,81$ $\mathrm{cmol}_{\mathrm{c}} \mathrm{kg}^{-1} ; \mathrm{H}^{+} \mathrm{Al}^{\mathrm{c}+}$ (acidez potencial $)=$ $5,42 \mathrm{cmol} \mathrm{kg}^{-1} ; \mathrm{K}=0,20 \mathrm{mg} \mathrm{kg}^{-1} ; \mathrm{P}=$ $3,3 \mathrm{mg} \mathrm{kg}^{-1}$ e $\mathrm{B}=0,48 \mathrm{mg} \mathrm{kg}^{-1}$ (Extrator $\mathrm{BaCl}_{2} .2 \mathrm{H}_{2} \mathrm{O} 10 \%$ em ebulição). $\mathrm{O}$ carbono orgânico foi determinado segundo Walkley \& Black (1934), sendo o teor obtido corrigido para matéria orgânica, conforme Jackson (1964), resultando em $33 \mathrm{~g} \mathrm{~kg}^{-1}$. Os teores de $\mathrm{Cu}$ e $\mathrm{Zn}$ no extrator Mehlich-1 foram 0,5 e 0,7 mg kg-1.

Efetuou-se a calagem com calcário dolomítico PRNT $=100 \%$ (relação $\mathrm{Ca}: \mathrm{Mg}=4: 1$, na dose de 4,2 $\mathrm{t} \mathrm{ha}^{-1}$ segundo o critério de saturação de bases, visando uma elevação a $85 \%$. Aplicouse uma adubação uniforme correspondente a $180 ; 10,9 ; 4,8 ; 6,3 ; 2,3$ e 0,195 $\mathrm{kg} \mathrm{ha}^{-1}$ de $\mathrm{K}_{2} \mathrm{O}, \mathrm{Mg}, \mathrm{Cu}, \mathrm{Zn}, \mathrm{B}$ e Mo respectivamente, nas formas de cloreto de potássio, sulfato de magnésio, sulfato de cobre, sulfato de zinco, bórax e molibdato de amônio respectivamente. Aos 45 dias após o plantio, aplicou-se $37 \mathrm{~kg} \mathrm{ha}^{-1}$ de $\mathrm{N}$ na forma de nitrato de amônio. Nestas condições e sem nova aplicação de composto de lixo, como prevenção ao aumento da condutividade elétrica no solo, avaliou-se a ação residual das aplicações a lanço de doses de fósforo (superfosfato triplo, $45 \%$ de $\mathrm{P}_{2} \mathrm{O}_{5}$ ), associadas com doses de composto de lixo proveniente da UECRB/DF, sobre a produção de raízes comercializáveis de cenoura cv. Brasília, cuja semeadura foi realizada em 03/04/ 97 e a colheita em 04/07/97. Conforme nos cultivos anteriores, considerou-se raízes comercializáveis aquelas com diâmetro e comprimento médios de 2,5 a $4 \mathrm{~cm}$ e de 10 a $20 \mathrm{~cm}$, respectivamente, livres de nematóides, rachaduras e bifurcações. Considerou-se produção comercializável ao peso dessas raízes por hectare.

$\mathrm{Na}$ época da colheita, e seguindo o delineamento experimental, tomou-se 6 plantas ao acaso dentro de cada parcela útil e fez-se a separação da parte aérea e das raízes para a determinação em laboratório de pesos fresco e seco. Ambos tecidos vegetais foram lavados em 
Produção comercializável e teores de $\mathrm{Cu}$ e Zn em cenoura em decorrência da ação residual de fósforo e composto de lixo em solo sob cerrado.

Tabela 1. Atributos químicos e físicos do composto de lixo da UECRB/DF, médias de três repetições. Brasília, Embrapa Hortaliças, 1997.

\begin{tabular}{|c|c|c|c|c|c|c|c|c|c|c|c|c|c|}
\hline $\mathrm{Cu}$ & $\mathrm{Fe}$ & Mn & $\mathrm{Zn}$ & $\mathrm{Sr}$ & $\mathbf{Y}$ & Cd & $\mathbf{V}$ & $\mathrm{Ni}$ & $\mathrm{Be}$ & Al & $\mathrm{Cr}$ & $\mathrm{Ba}$ & B \\
\hline 60,5 & 71.660 & 240 & 890 & 315 & 10 & 6 & 177 & 124 & 1 & 54.566 & 256 & 564 & 22 \\
\hline \multicolumn{2}{|c|}{ 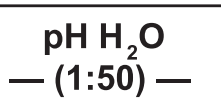 } & $\begin{array}{c}\mathrm{MO}^{*} \\
\left(\mathrm{~g} \mathrm{~kg}^{-1}\right)\end{array}$ & $\mathrm{C} / \mathrm{N}$ & $\mathbf{N}$ & $\mathrm{C}$ & $\mathbf{P}$ & $\mathrm{Na}$ & $\begin{array}{l}K \\
\%\end{array}$ & $\mathrm{Ca}$ & Mg & $S$ & $\begin{array}{l}\mathbf{H g} \\
-\mu \mathbf{g ~ k g}\end{array}$ & ${ }_{-1} \mathrm{~Pb}$ \\
\hline \multicolumn{2}{|c|}{$8,9 \pm 0,1$} & 41,6 & $13,8 / 1$ & 1,11 & 15,3 & 0,48 & 0,62 & 0,85 & 3,3 & 0,45 & 0,21 & 842 & 117 \\
\hline \multicolumn{2}{|c|}{$\begin{array}{c}\text { Densidade } \\
\left(\mathrm{g} \mathrm{cm}^{-3}\right)\end{array}$} & \multicolumn{3}{|c|}{ Retenção de água } & \multicolumn{3}{|c|}{ Umidade } & \multicolumn{3}{|c|}{ Inertes } & \multicolumn{3}{|c|}{$\begin{array}{l}\mathrm{DBO}_{5} \\
\left(\mathrm{mg} \mathrm{g}^{\circ}\right.\end{array}$} \\
\hline \multicolumn{2}{|c|}{0,88} & \multicolumn{3}{|c|}{54} & \multicolumn{3}{|c|}{15,1} & \multicolumn{3}{|c|}{33} & \multicolumn{3}{|c|}{275} \\
\hline
\end{tabular}

* Matéria orgânica

água deionizada, seguido de pesagem e secagem em estufa com circulação forçada de ar aproximadamente a $65^{\circ} \mathrm{C}$ até peso constante, quando determinou-se o peso da matéria seca. O material vegetal foi moído em moinho tipo Wiley e passado em peneira de 20 "mesh" $(0,841 \mathrm{~mm})$ de abertura. As amostras assim obtidas foram digeridas em uma mistura nitro-perclórica conforme Sarruge \& Haag (1974). No extrato obtido foram determinados $\mathrm{Cu}$ e $\mathrm{Zn}$ em um espectrofotômetro de absorção atômica marca Shimadzu AA670 G.

Os teores máximos de $\mathrm{Cu}$ e $\mathrm{Zn}$ determinados na matéria seca foram convertidos para matéria fresca e comparados com os permitidos em alimentos in natura pela legislação brasileira de alimentos (ABIA, 1985).

Por ocasião da primeira e da segunda aplicação de composto de lixo, foram retiradas amostras representativas desse resíduo conforme Kiehl \& Porta (1980), para processamento de análises químicas. Os teores médios de metais pesados determinados nessas amostras (Tabela 1), foram comparados com os permitidos em alguns países segundo Xin Tao et al. (1992).

\section{RESULTADOS E DISCUSSÃO}

No primeiro cultivo, o teste $\mathrm{F}$ indicou efeito linear para doses de fósforo e de composto de lixo em relação à produção comercializável de raízes de cenoura cv. Brasília, cuja máxima estimada foi de $37,02 \mathrm{t} \mathrm{ha}^{-1}$, correspondente às doses calculadas de $800 \mathrm{~kg}$ de $\mathrm{P}_{2} \mathrm{O}_{5} \cdot \mathrm{ha}^{-1}$ e de 49,2 t ha ${ }^{-1}$ de composto de lixo (Mesquita Filho, 1997). Por ocasião da

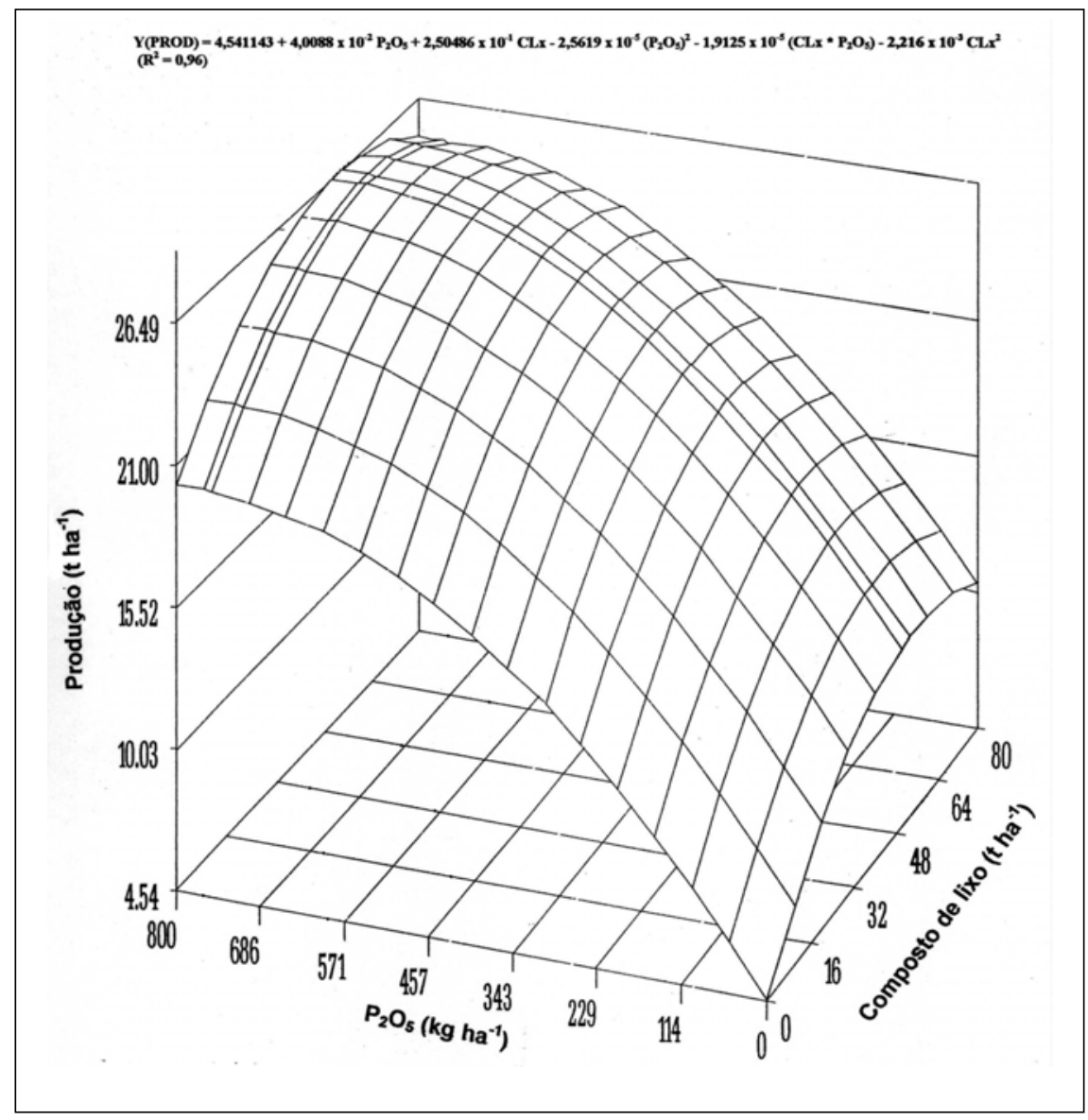

Figura 1. Superfície de resposta de cenoura cv. Brasília em decorrência da ação residual de diferentes doses de $\mathrm{P}_{2} \mathrm{O}_{5}$ e de composto de lixo, aplicadas a um LEd argiloso sob cerrado. Embrapa Hortaliças, Brasília-DF, 1997.

colheita, na solução do solo correspondente aos tratamentos, $0 ; 20 ; 40 ; 60$ e 80 tha ${ }^{1}$ de composto de lixo os valores de $\mathrm{pH}$ eram 5,$52 ; 5,59 ; 6,12 ; 6,25$ e 6,34 , e os de condutividade elétrica 0,$05 ; 1,8 ; 2,3 ; 3,1 \mathrm{e}$ $3,5 \mathrm{dS} \mathrm{m}^{-1}$ respectivamente (observação pessoal feita pelo primeiro autor).

No segundo cultivo, a análise estatística dos dados para produção de raízes comercializáveis revelou efeito significa- tivo $\mathrm{F}(\mathrm{p}<0,05)$ para doses residuais de fósforo e de composto de lixo. A produção comercializável máxima estimada de cenoura cv. Brasília foi de 32,2 tha ${ }^{-1}$, correspondente às doses calculadas de 800 $\mathrm{kg}$ de $\mathrm{P}_{2} \mathrm{O}_{5}$.ha- $\mathrm{ha}^{-1}$ e de 46,1 $\mathrm{t} \mathrm{ha}^{-1}$ de composto de lixo (Mesquita Filho, 1998).

Por ocasião da segunda colheita, verificou-se que na solução do solo dos tratamentos, 0; 20; 40; 60 e 80 t ha $^{-1}$ de 
Tabela 2. Teores de metais pesados em compostos de lixo urbano de alguns países, com base no peso do material seco.

\begin{tabular}{|c|c|c|c|c|c|c|}
\hline \multirow{3}{*}{ Elemento } & \multicolumn{6}{|c|}{ Países } \\
\hline & EUA & EUA & Itália & Espanha & França & Holanda \\
\hline & \multicolumn{6}{|c|}{ mg.kg ${ }^{-1}$} \\
\hline $\mathrm{Cu}$ & 100 & 200 & 422 & 200 & 250 & 630 \\
\hline $\mathrm{Ni}$ & - & - & - & 0,76 & 190 & 110 \\
\hline $\mathrm{Mn}$ & 1500 & 500 & 875 & 700 & 1000 & 1650 \\
\hline B & - & - & - & 3 & 60 & 60 \\
\hline $\mathrm{Hg}$ & - & - & - & - & 4 & 5 \\
\hline $\mathrm{Pb}$ & - & - & $6-5$ & 9 & 600 & 900 \\
\hline $\mathrm{Cd}$ & - & 100 & 8 & 0,04 & 7 & 6 \\
\hline $\mathrm{Cr}$ & - & - & 215 & 2 & 270 & 220 \\
\hline
\end{tabular}

Fonte: Adaptada de XIN et al. (1992)

(-) Dados não fornecidos

composto de lixo, os valores de $\mathrm{pH}$ eram 5,$54 ; 5,70 ; 5,85 ; 6,62$ e 6,77 , e os de condutividade elétrica 0,$07 ; 2,5 ; 3,0 ; 3,6$ e $4,3 \mathrm{dS} \mathrm{m}^{-1}$ respectivamente (observação pessoal feita pelo primeiro autor).

Um mês antes da segunda semeadura, foram aplicadas a lanço e incorporadas ao solo na profundidade $0-20 \mathrm{~cm}$, as mesmas doses de composto de lixo às parcelas correspondentes. Face ao elevado valor da condutividade elétrica $\left(4,3 \mathrm{dS} \mathrm{m}^{-1}\right)$ observado nas parcelas que receberam duas aplicações de $80 \mathrm{t} \mathrm{ha}^{-1}$, foi feito então trimestralmente, no período de 01/96 a 12/96, num total de 4 avaliações, o monitoramento do $\mathrm{pH}$ e da condutividade elétrica em todas as parcelas do experimento. Na época seca (agosto/setembro/96), toda área recebeu uma vez por semana irrigação por aspersão com lâmina líquida média de 3 mm.dia ${ }^{-1}$.

De acordo com a Tabela 1, o composto de lixo utilizado estava devidamente curado (Kiehl \& Porta, 1980). O manejo do solo realizado no período agosto/setembro/96, contribuiu para o abaixamento do $\mathrm{pH}$ e da condutividade elétrica do solo. Bernstein \& Ayres (1953), observaram redução de 50\% na produção de raízes de cenoura quando a condutividade elétrica do solo era de $4,6 \mathrm{dS} \mathrm{m}^{-1}$.

Os teores médios de $\mathrm{Cu}$ e $\mathrm{Zn}$ determinados no extrator Mehlich 1 antes do plantio foram 0,5 e 0,7 mg. $\mathrm{kg}^{-1}$ respectivamente, situando-se abaixo do nível crítico para solos sob cerrado, que segun- do Lopes (1983) é de $1 \mathrm{mg} . \mathrm{kg}^{-1}$, por este motivo, esses nutrientes foram inseridos por ocasião da adubação uniforme, a qual é considerada suficiente para a cultura da cenoura (Trani et al.,1993).

Para produção total de raízes de cenoura houve efeito linear e quadrático significativo $(p<0,01)$ para fósforo e composto de lixo. A interação linear $\mathrm{P}$ $\mathrm{x}$ quadrática composto de lixo foi significativa $(\mathrm{p}<0,01)$. Aos dados de produção ajustou-se a função Y(PROD) = $4,541143+4,0088 \times 10^{-2} \mathrm{P}_{2} \mathrm{O}_{5}+2,50486$ x $10^{-1} \mathrm{CLx}-2,5619 \times 10^{-5}\left(\mathrm{P}_{2} \mathrm{O}_{5}\right)^{2}-$ $1,9125 \times 10^{-5}\left(\mathrm{CLx} * \mathrm{P}_{2} \mathrm{O}_{5}\right)-2,216 \mathrm{x}$ $10^{-3} \mathrm{CLx}^{2}\left(\mathrm{R}^{2}=0,96\right)$, onde CLx $=$ composto de lixo (Figura 1). A produção total máxima estimada de raízes foi de $26,5 \mathrm{t} \mathrm{ha}^{-1}$, correspondente a uma produção comercial de $18,5 \mathrm{t} \mathrm{ha}^{-1}$, correlacionada com as doses de 762,5 $\mathrm{kg} \mathrm{ha}^{-1}$ de $\mathrm{P}_{2} \mathrm{O}_{5}$ e 53,2 tha-1 de composto de lixo. Essa produção representa $78 \%$ da média atualmente obtida no Distrito Federal. Donde depreende-se que para um próximo cultivo de cenoura cv. Brasília neste solo, é aconselhável nova aplicação de matéria orgânica.

Os teores de $\mathrm{Cu}$ e de $\mathrm{Zn}$ na parte aérea e nas raízes foram influenciados pelo $\mathrm{pH}$, teores de argila do solo e doses do composto de lixo $(p<0,01)$, mas não pelas doses de fósforo. $\mathrm{A}$ dose de $800 \mathrm{~kg}$ de $\mathrm{P}_{2} \mathrm{O}_{5} \mathrm{ha}^{-1}$ associada com a de $80 \mathrm{t} \mathrm{ha}^{-1}$ de composto de lixo proporcionaram os teores máximos de $\mathrm{Cu}$ e de $\mathrm{Zn}$ detectados tanto no solo quanto na matéria seca da parte aérea e das raízes de cenoura.
No solo, esses teores foram 0,7 e 1,2 mg. $\mathrm{kg}^{-1}$ respectivamente. $\mathrm{Na}$ matéria seca da parte aérea e das raízes os teores de $\mathrm{Cu}$ foram 24,6 e 55,1 mg. $\mathrm{g}^{-1}$ e os de $\mathrm{Zn}$ nesses tecidos foram 67,4 e 29,2 mg. $\mathrm{g}^{-1}$ respectivamente. Quando transformados para matéria fresca de raízes, os teores de $\mathrm{Cu}$ corresponderam a 0,74

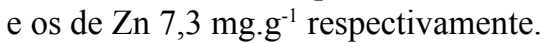

Teores na matéria seca de folhas compreendidos entre 29 - 39 mg. $\mathrm{g}^{-1}$ de $\mathrm{Cu}$ e 400 - 500 mg. $\mathrm{g}^{-1}$ de $\mathrm{Zn}$ são considerados fitotóxicos (Marschner, 1986). A exemplo dos dois primeiros cultivos, também neste não foram observados efeitos fitotóxicos causados por acúmulo desses elementos.

Costa et al. (1997), trabalhando em casa de vegetação, com doses crescentes de composto de lixo urbano aplicados a três tipos de solo, encontraram teores máximos de $2,83 \mathrm{mg} \mathrm{g}^{-1}$ de $\mathrm{Cu}$ e de $7,13 \mathrm{mg} \mathrm{g}^{-1}$ de Zn em matéria fresca de raízes de cenoura cv. Brasília. De acordo com esses autores, na matéria seca da parte aérea o teor máximo de 31,43 $\mathrm{mg} \mathrm{g}^{-1} \mathrm{de} \mathrm{Cu}$ foi considerado fitotóxico. De qualquer modo, tanto no experimento desses autores quanto no presente trabalho, os teores máximos de $\mathrm{Cu}$ e de $\mathrm{Zn}$ na matéria fresca das raízes, situaramse abaixo dos limites de tolerância (30 e $50 \mathrm{mg}$. $\mathrm{g}^{-1}$ respectivamente) em alimentos in natura (ABIA, 1985).

Nas condições do presente trabalho, observou-se que a cenoura cv Brasília é exigente em fósforo e matéria orgânica. Entretanto, é aconselhável cautela nas aplicações de composto de lixo em so- 
los semelhantes ao do presente trabalho, porque ainda se fazem necessários estudos de absorção sobre outros metais pesados nele existentes e que absorvidos por essa hortaliça, possam ser nocivos ao homem. Além disso aplicações indiscriminadas poderão provocar salinização no solo.

\section{AGRADECIMENTOS}

Os autores agradecem ao patrocínio da Fundação de Apoio à Pesquisa do Distrito Federal (FAPDF), assim como aos engenheiros agronômos Remidijo Tomazini Neto e Gilmar Paulo Henz, pelas sugestões na redação do texto. Aos laboratoristas Sarita Mazutti, Damião Fernandes da Cunha, Pedro Couto e Cristiano da Silva, pelas análises de solo e de tecido vegetal. Ao técnico agrícola João Lopes da Cruz, pelo auxílio prestado durante a condução do experimento.

\section{LITERATURA CITADA}

ABIA (São Paulo, SP). Compêndio da legislação de alimentos. São Paulo, 1985. v. 1, 185 p. ALVES, W.L.; MELO, W.J.; FERREIRA, M.E Estudo do composto de lixo urbano em um solo arenoso e em plantas de sorgo. Revista Brasileira de Ciência do Solo, Campinas, v. 23, n. 3, p. 729736, 1999.
BERNSTEIN, L.; AYRES, A.D. Salt tolerance of varieties of carrot. Proceedings of the American Society for Horticultural Science, v. 61, p. 360366, 1953.

CABRERA, F.; DIAZ, E.; MADRID, L. Effect of using urban compost as manure on soil contents of some nutrients and heavy metals. Journal of Food and Agriculture, v. 47, p.159-169, 1989.

COSTA, C.A.; CASALI, V.W.D.; LOURES, E.G.; CECON, P.R.; JORDÃO, C.P. Teor de metais pesados em alface (Lactuca sativa $\mathrm{L}$ ) adubada com composto de lixo urbano. Revista Ceres, Viçosa, v. 41, p. 629-640, 1994.

COSTA, C.A.; CASALI, V.W.D.; LOURES, E.G.; CECON, P.R.; JORDÃO, C.P. Teor de zinco, cobre e cádmio em cenoura em função de doses crescentes de composto de lixo urbano. Horticultura Brasileira, Brasília, v. 15, n. 1, p. 10-14, 1997.

CRAVO, M.S.; MURAOKA, T; GINÉ, M.F. Caracterização química de compostos de lixo urbano de algumas usinas brasileiras. Revista Brasileira de Ciência do Solo, Campinas, v. 22, n. 3, p. 547-553, 1998.

EMBRAPA. Serviço Nacional de Levantamento e Conservação de Solos (Rio de Janeiro, RJ) Manual de métodos de análises do solo. Rio de Janeiro, 1979. 73 p.

FORTES NETO; P. BALLESTERO, S.D.; FORTES, N.L.P.; GADIOLI, J. MONTEIRO, M.L.; ROMANO, F.C.; THIMOTEO, A.C.; INABA, R.M.; CEZAR, V.R.S.; CARNIERI, M. Efeito de doses crescentes de composto de lixo no solo e em algumas culturas olerícolas. In: REUNIIÃO BRASILEIRA DE FERTILIDADE DO SOLO E NUTRIÇÃO DE PLANTAS, 22., 1996, Manaus, AM. Resumos expandidos... Manaus: SBCS-FCA EMBRAPA-CPAO/INPA, 1996. p.417-418.
HE, X.; TRAINA, S.J.; LOGAN, T.J. Chemical properties of municipal solid waste composts. Journal of Environmental Quality, v. 21, p. 318 329, 1992.

JACKSON, M.L. Determinaciones de materia organica en los suelos. In: JACKSON, M.L., ed. Análisis químico de suelos. Barcelona: Omega, 1964. p. 282-310.

LOPES, A.S. Solos sob "cerrado": características, propriedades e manejo. Piracicaba: Potassa, 1983. 162 p.

KIEHL, E.J.; PORTA, A. Análise de lixo e composto. Piracicaba: ESALQ, 1980. 55 p.

MARSCHNER, H. Mineral nutrition of higher plants. London: Academic Press, 1986.

$674 \mathrm{p}$.

MESQUITA FILHO, M.V.; CRISÓSTOMO, L.A.; SILVA, T.G. Rendimento de cenoura em função da aplicação de nitrogênio e fósforo em solo sob cerrado. Horticultura Brasileira, Brasília, v. 3, n. 2, p. 39-40, 1985.

SARRUGE, J.R.; HAAG, H.P. Análises químicas em plantas. Piracicaba: ESALQ, 1974. p. 25-29.

TRANI, P.E.; FORNASIER, J.B.; LISBÃO, R.S. Nutrição mineral e adubação da cenoura. In: SIMPÓSIO SOBRE ADUBAÇÃO E NUTRIÇÃO DE HORTALIÇAS, 1990, Jaboticabal, SP. Nutrição e adubação de hortaliças: anais. Piracicaba: POTAFOS, 1993. p. 447-462.

WALKLEY, A.; BLACK, I.A. Examination of degtjareff method for determining soil organic matter and a proposed modification of the chromic acid titration. Soil Science, v. 37, p. 29-38, 1934. XIN, T.H.; TRAINA, S.J.; LOGAN, T.J. Chemical properties of municipal solid waste compost. Journal of Environmental Quality, v. 21, p. 318329, 1992. 